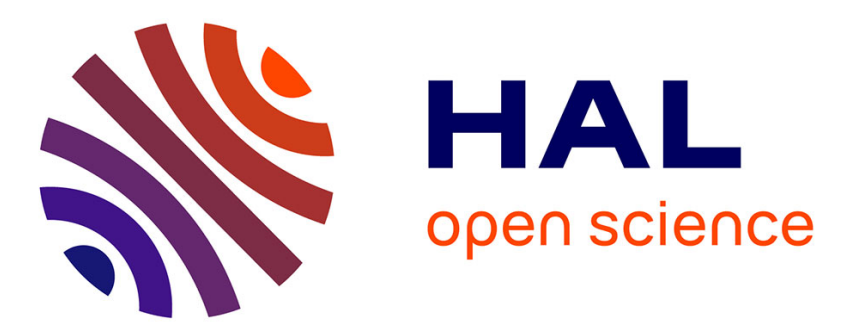

\title{
Dispersions of magnetic nanoparticles in the mixture ethyleneglycol-choline chloride: The role of solvent association
}

\author{
C. Kern, R. Aquino, Emeric Dubois, R. Perzynski, V. Peyre
}

\section{To cite this version:}

C. Kern, R. Aquino, Emeric Dubois, R. Perzynski, V. Peyre. Dispersions of magnetic nanoparticles in the mixture ethyleneglycol-choline chloride: The role of solvent association. Journal of Molecular Liquids, 2018, 268, pp.545-552. 10.1016/j.molliq.2018.07.063 . hal-01927141

\section{HAL Id: hal-01927141 \\ https://hal.sorbonne-universite.fr/hal-01927141}

Submitted on 19 Nov 2018

HAL is a multi-disciplinary open access archive for the deposit and dissemination of scientific research documents, whether they are published or not. The documents may come from teaching and research institutions in France or abroad, or from public or private research centers.
L'archive ouverte pluridisciplinaire $\mathbf{H A L}$, est destinée au dépôt et à la diffusion de documents scientifiques de niveau recherche, publiés ou non, émanant des établissements d'enseignement et de recherche français ou étrangers, des laboratoires publics ou privés. 


\title{
Dispersions of Magnetic Nanoparticles in the Mixture Ethyleneglycol-Choline Chloride : the Role of Solvent Association
}

\author{
C. Kern ${ }^{\mathrm{a}, \mathrm{b}, \mathrm{c}}$, R. Aquino ${ }^{\mathrm{b}, \mathrm{c}}$, E. Dubois ${ }^{\mathrm{a}}$, R. Perzynski ${ }^{\mathrm{a}}$, V. Peyre ${ }^{\mathrm{a}, *}$ \\ a Sorbonne Université, CNRS, Laboratoire Physico-Chimie des Electrolytes et des Nanosystèmes Interfaciaux, PHENIX, F-75005 Paris, France \\ ${ }^{b}$ Grupo de Fluidos Complexos and Instituto de Quimica, Universidade de Brasilia, CP 04478, 70904970 Brasilia (DF), Brazil \\ ${ }^{c}$ Faculdade UnB Planaltina, Universidade de Brasilia, 73345-010, Planaltina (DF), Brazil
}

\begin{abstract}
The mixture of choline chloride and ethyleneglycol ((1:3) on the molar scale) is first characterized using density, viscosimetry and refractive index measurements, in the temperature range $20-45^{\circ} \mathrm{C}$, with controlled amounts of water. Thermodynamic parameters such as the activation entropy, enthalpy and Gibbs energy for the viscous flow are deduced from the data and show that the solvent is highly structured. A protocol to disperse in this solvent $10 \mathrm{~nm}$ maghemite nanoparticles previously synthesized in water is described. The good quality of the dispersion is assessed by visual observations, optical microscopy, small angle X-ray scattering (SAXS), magnetization measurements, viscosimetry, and light scattering. The results lead to the description of a local viscosity around the particles, lower than the macroscopic one, that could be the result of the solvent segregation with ethyleneglycol being mostly present in the close vicinity of the particles.
\end{abstract}

\author{
Keywords: \\ Magnetic nanoparticles \\ Colloidal dispersions \\ Choline chloride \\ Ethyleneglycol \\ Maghemite
}

\section{Introduction}

Dispersions of nanoparticles (NPs) in ionic liquids (ILs) have drawn recently huge attention. The electrochemical and conducting properties of the ILs can indeed be combined with specific (electronic, magnetic, optical...) properties of the nanoparticles to obtain multiple stimuli responsive materials with possible applications in electrochemistry, synthesis, catalysis, separation fields $[1,2]$.

However, one serious drawback of such solvents is their price, toxicity and availability. For this reason, deep eutectic solvents (DES) and related complex solvents can be interesting alternatives. These liquids are made of a quaternary ammonium salt (choline chloride in our case) mixed with a molecular hydrogen bond donor (HBD). Since the molar ratio of ammonium salt in the final liquid is huge, the new solvent is closer to an ionic liquid than to an electrolyte solution. Indeed, the physicochemical properties of DESs such as negligible vapor pressure, thermostability, wide electrochemical potential windows, tunability are similar to those of the traditionally used ionic liquids. DESs can also have the ILs' drawbacks such as high viscosity [3]. Since the interest for DESs is more recent than that for standard ILs, data about both the DESs and dispersions of particles in DESs are still scarce $[4,5]$. The complexity of the solvent,

*Corresponding author : veronique.peyre@upmc.fr made of three components (the cation, the anion and the HBD) makes any prediction about colloidal stability in DESs impossible in the actual state of our knowledge. For this reason and due to the interesting properties of DESs such as cost and availability there is a huge need for experimental data about dispersions of NPs in DESs.

In this work, we use a solvent mixture made of choline chloride $(\mathrm{ChCl})$, a widely used, cheap, bio-sourced component, mixed with ethyleneglycol (EG) as HBD. Thanks to its composition, it is closely related to DES and is sometimes classified among them although it is not an eutectic mixture on the strict basis of melting temperatures. This solvent will be named ChEG hereafter. Though somehow toxic, EG is chosen since it can form with $\mathrm{ChCl}$ a liquid of low viscosity at room temperature (around $35 \mathrm{mPa} . \mathrm{s}$ at $20^{\circ} \mathrm{C}$ for $\mathrm{ChCl}: \mathrm{EG}(1: 3$ in mol:mol) compared to more than $1000 \mathrm{mPa}$.s for the widely used ChCl:urea (1:2)[6]). Based on the analysis of the conductivity and viscosity, it was shown [7] that $\mathrm{ChCl}$ : EG mixtures switch from a dilute salt solution behavior to an ionic liquid behavior for $\mathrm{ChCl}$ :EG composition about 1:4 $(20 \mathrm{~mol} \%=36$ vol\% of $\mathrm{ChCl}$ ). For this reason, a composition slightly higher $(1: 3-25 \mathrm{~mol} \%=43 \mathrm{vol} \%$ of $\mathrm{ChCl})$ is used in this work.

A dispersion of NPs in ChEG could be obtained by direct synthesis in the complex solvent. This leads to NPs with surface properties different from the synthesis in water and to enhanced catalytic properties $[8,9]$. However, the nature of the surface 
is not wery well understood and controlled. Morever, it can be difficult to eliminate the side-products of the NPs synthesized in ChEG.

Here, we chose a NPs synthesis in an aqueous medium which is easier, cheaper and already well known. Therefore, a transfer to ChEG after a synthesis in water appears more advantageous, all the more so since the interface NPs-solvent can be controlled and adapted during this step. The chosen nanoparticles are iron oxide maghemite particles (diameter $\approx 10 \mathrm{~nm}$ ), synthesized by coprecipitation in alkaline aqueous medium $[10,11,12]$. Their surface state can be tuned to monitor the surface charge by changing the $\mathrm{pH}$, or by adsorption of various ligands such as citrate or polyacrylate. Such particles have already been successfully dispersed in an ionic liquid, ethylammonium nitrate $[11,13,14,15]$. The particles being magnetic, the observed stable dispersion is indeed a ferrofluid (FF). The best colloidal stability was obtained for particles coated with citrate or poly(acrylate), using $\mathrm{Na}^{+}$as a counterion $[11,15]$. The stability was ensured by the formation of layers of solvent ions around the particles, providing a steric hindrance to particles approach [14]. The determining effect of small amounts of water was also evidenced, leading to enhanced flocculation by destructuration of these protective layers [14].

In the present work, we transfer similar citrate-coated NPs $\left(\mathrm{Na}^{+}\right.$counterions) from water to ChEG. In the first part, we detail the samples and techniques used. We then analyse the solvent - mixture ChCl-EG (1:3) - by measuring viscosity, density and refractive index in the temperature range $20-45^{\circ} \mathrm{C}$, as a function of the water content. These parameters are indeed necessary for the analysis of the obtained colloidal dispersions in ChEG. A model to describe the results is proposed. This solvent is subsequently used to disperse maghemite nanoparticles. We assess the colloidal stability by means of visual observations, optical microscopy, small angle X-ray scattering (SAXS), magnetization measurements, viscosimetry, and dynamic light scattering. Finally, we discuss the results and conclude on the possible influence of a local viscosity different from the macroscopic one to understand the data.

\section{Materials and methods}

The following products are purchased and used as received: choline chloride (Sigma Aldrich); ethyleneglycol (Normapur, VWR); nitric acid (69.5\% water solution, Carlo Erba); hydrochloric acid (37\% water solution, AnalaR Normapur, VWR); citric acid $\left(\mathrm{H}_{3} \mathrm{Cit}\right.$, Rectapur, Prolabo); iron(II) chloride (AnalaR Normapur, VWR); iron(III) chloride (Prolabo); iron(III) nitrate (Technical, VWR); trisodium citrate ( $\mathrm{Na}_{3} \mathrm{Cit}$, Merck); acetone (technical, VWR); diethyl ether (99.8\% pur., AnalaR Normapur).

Water content is analyzed using coulometric Karl Fisher titration (Schott TitroLine KF Trace). Density is measured thanks to an Anton Paar DSA 5000M, with precision $10^{-6}$ $\mathrm{g} / \mathrm{mL}$ and temperature accuracy better than $10^{-3}{ }^{\circ} \mathrm{C}$. Viscosity of ChEG and ChEG/water mixtures is determined using an Anton Paar Automated MicroViscometer (AMVn) instrument by using the falling ball model in a $1.8 \mathrm{~mm}$ (resp. $3.0 \mathrm{~mm}$ ) capillary with a $1.5 \mathrm{~mm}$ (resp $2.5 \mathrm{~mm}$ ) falling ball. The instrument is first calibrated with reference standard oils N14 (resp. N44) from Cannon Instrument Company provided by Anton Paar ( $\eta=71.29 \mathrm{mPa} \cdot \mathrm{s}$ (resp. $19.68 \mathrm{mPa} \cdot \mathrm{s}), \rho=$ $0.8249 \mathrm{~g} / \mathrm{mL}$ (resp. $0.8092 \mathrm{~g} / \mathrm{mL}$ ) at $25^{\circ} \mathrm{C}$ )). Dynamic light scattering is performed on a Vasco instrument from Cordouan Technologies, operating at $\lambda=656 \mathrm{~nm}$, with a temperature control better than $0.1^{\circ} \mathrm{C}$. The instrument uses a very thin film (around $200 \mu \mathrm{m}$ ) and a backscattering detection at $\theta=$ $135^{\circ}$, which avoids multiple scattering and allows measurements in optically absorbing samples such as ferrofluids. The corresponding scattering vectors $Q$ values are $Q=2.3$ $10^{-3} \AA^{-1}$ in water and $2.510^{-3} \AA^{-1}$ in ChEG. The index of refraction is measured with a refractometer Arago from Cordouan Technologies at $656 \mathrm{~nm}$, with a temperature control better than $0.1^{\circ} \mathrm{C}$. The samples are observed on the micron scale with an optical microscope (Olympus BX51 with lens 40x/0.6 and camera $\times 5$ ). Small angle X-rays scattering (SAXS) is performed at the SWING beam line of synchrotron Soleil (France). Two different configurations are used, giving access to the Q-range $2.10^{-3} \AA^{-1}<\mathrm{Q}<0.4 \AA^{-1}$. The beam energy of $7 \mathrm{keV}$ (corresponding to $\lambda=1.77 \AA$ ) is chosen to avoid $\mathrm{X}$-ray absorption by iron. Standard correction procedures are applied for sample volume, empty cell signal subtraction and detector efficiency to obtain the scattered intensity in absolute scale $\left(\mathrm{cm}^{-1}\right)$. Such data reduction is done using the software Foxtrot ${ }^{\circledR}$. Magnetization is registered using a home-made Vibrating Sample Magnetizer (VSM [16, 17]). The stability under magnetic field is checked from the absence of diffraction pattern when the sample is illuminated by a He-Ne laser under the field of an electromagnet. Above the threshold of stability, a diffracted line appears in the direction perpendicular to the magnetic field due to the formation of concentrated phase needles resulting from a phase separation [18].

\section{Choline Chloride-Ethyleneglycol (ChEG)}

The complex solvent chosen here, based on choline chloride $(\mathrm{ChCl})$ and ethyleneglycol (EG) is prepared by simple mixture of the two components at $60^{\circ} \mathrm{C}$ in closed bottles in order to avoid water uptake. No liquid mixture could be observed for a molar ratio $\mathrm{ChCl}$ :EG of composition $(1: 1)(50 \mathrm{~mol} \%)$ even at high temperature and crystals appeared upon cooling for the ratio $(1: 2)(33 \mathrm{~mol} \%)$. For this reason and according to Abbott's criterion to obtain an ionic liquid behaviour [7], we chose to work with a molar ratio (1:3). The properties of both components and resulting solvent are given in Table 1 . The amount of water in the prepared solvent, determined from Karl Fisher titration, is $0.2 \mathrm{w} \%$.

Only few data are available in literature (see Table 1), with scattered values. Our density is close to the results of [7]. However, the viscosity we obtain is much higher. Since the water contents can be of importance for the viscosity, we investigate the density and viscosity of the ChEG, in the temperature range $20-45^{\circ} \mathrm{C}$ (Figure 1 and 2, data in SI) for various quantities of 


\begin{tabular}{|c|c|c|c|}
\hline Properties & $\begin{array}{c}\text { Ethylene } \\
\text { glycol (EG) }\end{array}$ & $\begin{array}{c}\text { Choline } \\
\text { Chloride }(\mathrm{ChCl})\end{array}$ & $\begin{array}{c}\text { ChCl:EG stor } \\
1: 3(\mathrm{ChEG})\end{array}$ \\
\hline $\mathrm{M}(\mathrm{g} / \mathrm{mol})$ & 62.07 & 139.63 & 81.46 in \\
\hline Melting T $\left({ }^{\circ} \mathrm{C}\right)$ & $-13[19]$ & $247[20]$ & $-8[21]$ \\
\hline Boiling T $\left({ }^{\circ} \mathrm{C}\right)$ & $197.3[19]$ & decomposition & \\
\hline Density & $1.10988[22]$ & $1.1[20]$ & $1.1367[21]$ \\
$\left(\mathrm{g} / \mathrm{cm}^{3}\right)$ & $1.11004[23]$ & (for 70w\% & $1.118[7]$ \\
& & in water) & $1.115167^{*}$ \\
\hline Viscosity & $17.13[24]$ & - & $26[21]$ \\
$\left(10^{-3} \mathrm{~Pa} / \mathrm{s}\right)$ & & & $19[7]$ \\
& & & $31.6^{*}$ \\
\hline Refractive & $1.4318\left(20^{\circ} \mathrm{C}\right)$ & - & $1.456^{*}$ \\
index & {$[19]$} & & \\
\hline Molar volume & $55.92[22]$ & $127 \pm 5[20]$ & $71.66[21]$ \\
$\left(\mathrm{cm}^{3} / \mathrm{mol}\right)$ & & & $72.86[7]$ \\
& & & $72.53^{*}$ \\
\hline
\end{tabular}

Table 1: Properties of the individual components of ChEG and of ChEG at $\mathrm{T}=25^{\circ} \mathrm{C}$ except when another temperature is specified. $*=$ this work

added water. Unless a huge amount of water is present in literature samples, this cannot be the reason for the observed viscosity discrepancies ${ }^{1}$.

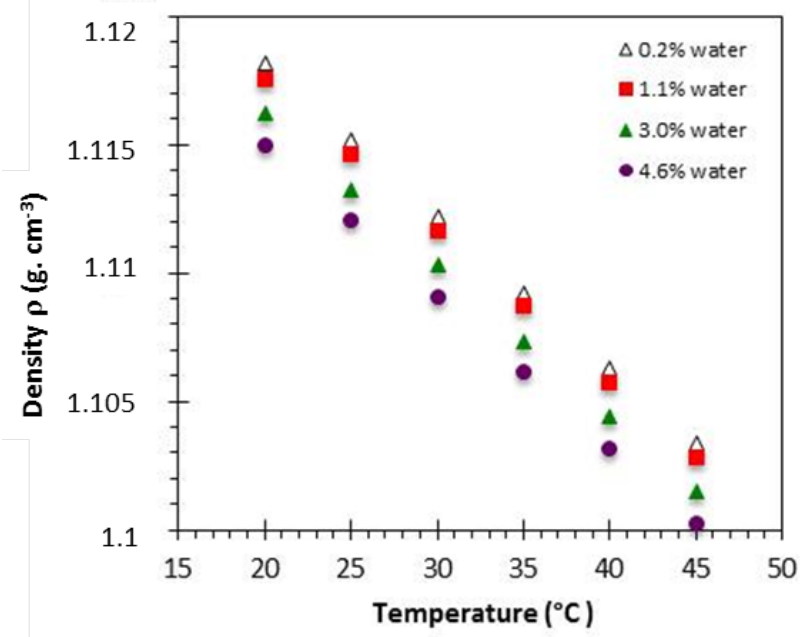

Figure 1: Density versus temperature for the solvent ChCl:EG (1:3) with different water contents (in weight percent).

The evolution of the viscosity with the water content of Fig. 2 shows the importance of controlling this parameter. Indeed these solvents are hygroscopic. We left a vial open to the ambient air and recorded the mass increase, attributed to atmospheric water uptake, for several days. The water uptake is very low $(+0.24 \mathrm{w} \%)$ during the first four hours. It is thus possible to work quickly with short opening of the containers for the necessary handling of ChEG. In the long run, the water uptake is $+13 \mathrm{w} \%$ after 3 weeks. Thus the solvent must be

\footnotetext{
${ }^{1}$ Another reason for the low viscosity values found in the literature could be a degradation of the solvent, for example due to a heating at too high temperature, as this solvent presents a thermal decomposition above $100^{\circ} \mathrm{C}$ [25]
}

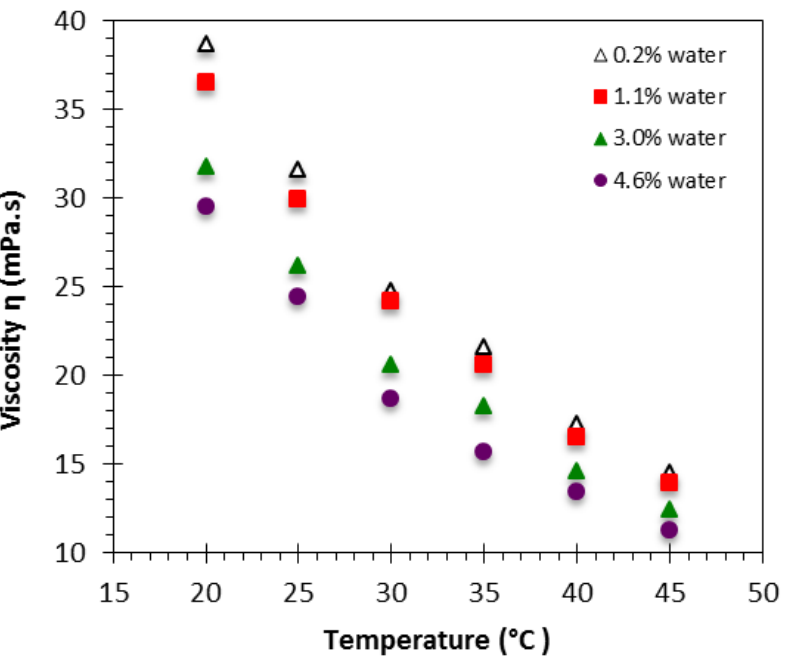

Figure 2: Viscosity versus temperature for the solvent ChCl:EG (1:3) with different water contents (in weight percent).

\subsection{Dependence of the density on the water-content as a function of temperature}

Molar volume of the solvent (here, wet ChEG) $\mathrm{V}_{\text {slvt }}$ can be calculated from experimental density values $\rho$ for each temperature according to :

$$
V_{s l v t}=M_{s l v t} / \rho
$$

( $\mathrm{M}_{\text {slvt }}$ is the molar mass of the solvent)

The quantity of water is taken into account in $\mathbf{M}_{\text {slvt }}$ through:

$$
M_{s l v t}=(1-x) M_{C h E G}+x M_{H_{2} O}
$$

where $x$ is the molar fraction of water deduced from KarlFisher titration, $\mathrm{M}_{C h E G}$ and $\mathrm{M}_{\mathrm{H}_{2} \mathrm{O}}$ are, respectively, the molar mass of the pure ChEG (table 1) and water. $\mathrm{V}_{\text {slvt }}$ vs water content $x$ is plotted in supporting information (Fig. SI-1), for temperatures between $T=20^{\circ} \mathrm{C}$ and $45^{\circ} \mathrm{C}$. The plots are linear and can be approximated for each $T$ by :

$$
V_{s l v t}=V_{C h E G}^{*}+x\left(V_{H_{2} O}^{\infty}-V_{C h E G}^{*}\right)
$$

where $\mathrm{V}_{\mathrm{H}_{2} \mathrm{O}}^{\infty}$ is the partial molar volume of water at infinite dilution and $\mathrm{V}_{C h E G}^{*}$ is the molar volume of pure (dry) ChEG, at the given temperature $T$. The y-intercept is thus $\mathrm{V}_{C h E G}^{*}$ at $T$. From the slopes of the curves, one can calculate $\mathrm{V}_{\mathrm{H}_{2} \mathrm{O}}^{\infty}$ as a function of $T$. Table 2 reports these $\mathrm{V}_{C h E G}^{*}$ and $\mathrm{V}_{H_{2} O}^{\infty}$ as well as that of the molar volume of pure water $\mathrm{V}_{\mathrm{H}_{2} \mathrm{O}}^{*}$ calculated from [26], in the temperature range explored. $\mathrm{V}_{\mathrm{H}_{2} \mathrm{O}}^{\infty}$ is smaller by $1 \mathrm{~cm}^{3}$ compared to $\mathrm{V}_{\mathrm{H}_{2} \mathrm{O}}^{*}$ at $20^{\circ} \mathrm{C}$ and stays small whatever the temperature. 
The same decrease was observed for water dissolved in pure EG [27] and can be attributed to a more efficient packing of water in the mixture with less $\mathrm{H}$ bonds around $\mathrm{H}_{2} \mathrm{O}$ that in bulk water.

\begin{tabular}{|c|c|c|c|c|c|c|}
\hline $\mathrm{T}$ & 20 & 25 & 30 & 35 & 40 & 45 \\
\hline $\mathrm{V}_{C h E G}^{*}$ & 72.840 & 73.035 & 73.230 & 73.425 & 73.620 & 73.817 \\
\hline $\mathrm{V}_{\mathrm{H}_{2} \mathrm{O}}^{\infty}$ & 16.994 & 17.083 & 17.174 & 17.266 & 17.361 & 17.487 \\
\hline $\mathrm{V}_{\mathrm{H}_{2} \mathrm{O}}^{*}$ & 18.032 & 18.053 & 18.079 & 18.108 & 18.141 & 18.178 \\
\hline
\end{tabular}

Table 2: Molar volume of pure ChEG $V_{C h E G}^{*}\left(\mathrm{~cm}^{3} / \mathrm{mol}\right)$, partial molar volume of water at infinite dilution in the ChEG-water mixture $V_{H 2 O}^{\infty}$, and molar volume of pure water $V_{H 2 O}^{*}$ for various temperature T $\left({ }^{\circ} \mathrm{C}\right)$. Assuming an ideal mixture, $\mathrm{V}_{C h E G}^{*}$ is consistent with a molar volume of $72.0+/-1.5 \mathrm{~cm}^{3} / \mathrm{mol}$ at $20^{\circ} \mathrm{C}$ which can be estimated from the pure component volumes with an imprecise density of choline chloride.

The expansion of $\mathrm{V}_{s l v t}$ with temperature can be quantified with the volumetric isobaric thermal expansion coefficient, defined as $\alpha_{P}=\left(1 / V_{s l v t}\right)\left(\partial V_{s l v t} / \partial T\right)_{P}$. The coefficient $\alpha_{P}$ values are between $5.2710^{-4}$ and $5.3610^{-4} \mathrm{~K}^{-1}$ in the temperature range explored whatever the water content. These values are close to those determined for the mixture $\mathrm{ChCl} / \mathrm{EG}$ (1:2) (5-6 $\left.10^{-4} \mathrm{~K}^{-1}\right)$ [28] and a bit smaller than the one of EG (6.4 $10^{-4} \mathrm{~K}^{-1}$ [24]). This indicates that ChEG is a slightly more associated liquid than pure EG.

\subsection{Dependence of viscosity on the water-content as a function of temperature}

The temperature-dependence of macroscopic viscosities $\eta$ of $\mathrm{ChCl}$ :EG (1:3) mixtures (Fig. 2) can be described with Eyring's model [29] for each water content as:

$$
\eta=h N_{A} / V_{s l v t} \exp \left(\Delta G_{a} / R T\right)
$$

with $h$, the Planck constant, $N_{A}$, the Avogadro number and $\Delta G_{a}$ the activation Gibbs energy for the viscous flow. From equation 4, $\Delta G_{a}$ is obtained for each $x$ and $T$ using the experimental $\eta$ and $V_{\text {slvt }}$.

In a first step, let us analyse $\Delta G_{a}$ at $298 \mathrm{~K}$ as a function of the water molar fraction $x$. We obtain a linear function, which can be described with $\Delta G_{a}=-22,9 x+21,5(\mathrm{~kJ} / \mathrm{mol})$. The y-intercept $(21.5 \mathrm{~kJ} / \mathrm{mol})$ corresponds to $\Delta G_{a}$ value for pure (dry) ChEG. The presence of water lowers the activation Gibbs energy for the flow, i.e., the energy barrier to overcome for the fluid to flow is smaller. This reduction of $\Delta G_{a}$ can originate either from a smaller enthalpy contribution (intermolecular interactions in the fluid are smaller) or from an entropic contribution (more configurations are accessible) during the flow process according to Eyring's hole theory for viscosity.

In a second step, the analysis of $\Delta G_{a}$ as a function of $T$ for each water content gives the activation flow enthalpy and entropy from $\Delta G_{a}=\Delta H_{a}-T \Delta S_{a}$. When a molecule is on its way to change location (producing a flow), it must overcome an energy barrier made of two contributions. First, it must fight against the loss of interaction energy with its neighbours of the initial place : this is quantified as $\Delta H_{a}$. Second, since it is not linked any more to its neighbours, it gains rotation entropy. This

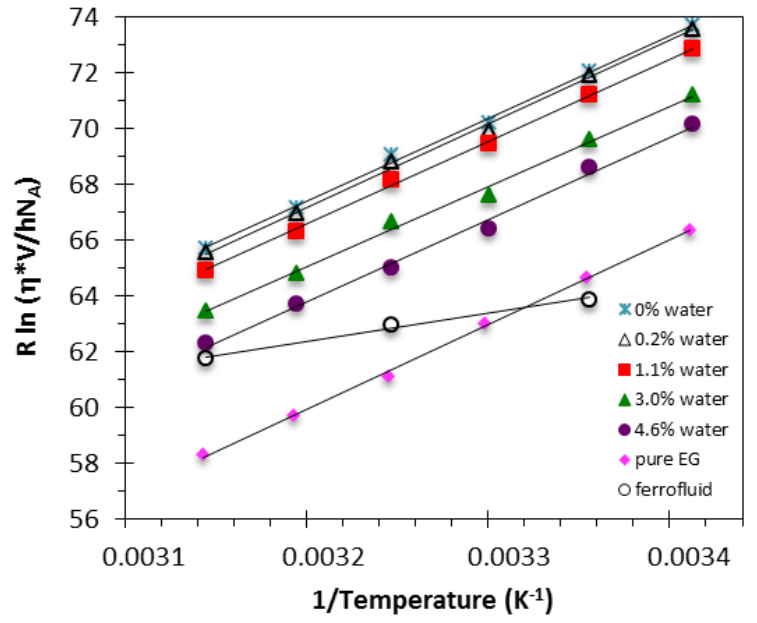

Figure 3: Eyring's plot for the solvent $\mathrm{ChCl}$ :EG (1:3) with different water contents (in weight percent). $0 \%$ was obtained using extrapolated values of molar volumes and viscosities to null water content. Values for pure EG are from [24]. For the ferrofluid, the molar volume is that of the driest ChEG (0.2\% water) with the local viscosity discussed in the text.

is $\Delta S_{a}$. The flow will be easier if its activation energy $\Delta G_{a}$ is low, e.g., if $\Delta H_{a}$ and $-T \Delta S_{a}$ are low (high $\Delta S_{a}$ ). These contributions can be determined according to equation (4) supposing $\Delta H_{a}$ and $\Delta S_{a}$ are independent of T. $R \ln \left[\eta V_{s l v t} / h N_{A}\right]=\Delta G_{a} / T$ plotted vs $(1 / T)$ (Eyring plot, figure 3$)$ is a straight line which gives access to $\Delta H_{a}$ (slope) and $\Delta S_{a}$ (y-intercept) (Table 3). This is applied for each water content as well as for the extrapolated "pure dry solvent" using $V_{C h E G}^{*}$ and viscosity data linearly extrapolated to zero water content (data in SI). All $\Delta H_{a}$ values are scattered around the mean value $29.5 \pm 0.5 \mathrm{~kJ} / \mathrm{mol}$, close to the enthalpy of activation of pure EG $(30.0 \mathrm{~kJ} / \mathrm{mol})[24]$. This implies that, for the viscous flow, the formation of an activated species in the solvent made of $\mathrm{ChCl}$ and $\mathrm{EG}$ is probably related to the same kind of intermolecular forces as in pure EG. The presence of some water does not deeply modify this interaction energy. It is coherent with a view of the cohesion of ChEG due to $\mathrm{H}$ bonds between all three components (EG, $\mathrm{ChCl}$ and water).

$\Delta S_{a}$ values are scattered around $28.5 \pm 1.5 \mathrm{~J} \mathrm{~mol}^{-1} \mathrm{~K}^{-1}$ (or - $T \Delta S_{a}=-8.5 \pm 0.6 \mathrm{~kJ} \mathrm{~mol}^{-1}$ at $298 \mathrm{~K}$ ) for all water contents. The entropic contribution $-T \Delta S_{a}$ to $\Delta G_{a}$ is negative and is about one third of $\Delta H_{a}$ as it is also observed for pure EG $\left(-T \Delta S_{a}=\right.$ $-10.7 \mathrm{~kJ} \mathrm{~mol}^{-1}$ at $298 \mathrm{~K}$ [24]). The entropy term is high and lowers the $\Delta G_{a}$ value. However, the solvent, i.e. the mixture of $\mathrm{EG}$ and $\mathrm{ChCl}$, is still a structured solvent that needs to locally destructurate in order to flow, as pure EG. A more detailed analysis of the contributions of $-T \Delta S_{a}$ and $\Delta H_{a}$ to $\Delta G_{a}$ with the water content in ChEG is delicate since a small experimental imprecision on the slope determination in Figure 3 (i.e., $\Delta H_{a}$ ) has a huge impact on the y-intercept $\Delta S_{a}$.

This set of data on the ChCl:EG (1:3) mixture provides a good picture of this new solvent, scarcely described in the literature, here studied within several conditions of water uptake. Its stability in the long run for $\mathrm{T}<30^{\circ} \mathrm{C}$ was also verified by 


\begin{tabular}{|c|c|c|c|}
\hline & ChEG (1:3) * & $\begin{array}{c}\text { Pure EG } \\
{[24]}\end{array}$ & FF in ChEG * \\
\hline $\begin{array}{c}\Delta G_{a}\left(\mathrm{~kJ} . \mathrm{mol}^{-1}\right) \\
(298 \mathrm{~K})\end{array}$ & 21.4 & 19.3 & 19 \\
\hline$\Delta H_{a}\left({\left.\mathrm{~kJ} . \mathrm{mol}^{-1}\right)}^{-1}\right)$ & 29.9 & 30.0 & 10.0 \\
\hline $\begin{array}{c}-\mathrm{T} \Delta S_{a}\left(\mathrm{~kJ}^{\prime} \mathrm{mol}^{-1}\right) \\
(298 \mathrm{~K})\end{array}$ & -8.5 & -10.7 & 9.0 \\
\hline
\end{tabular}

Table 3: Flow activation Gibbs energy, enthaly and entropy derived from viscosity for the ChEG solvent with $0.2 \%$ water, for EG and for the ferrofluid in ChEG. * this work

regular checking of the samples over several months.

\section{Dispersions in ChEG}

The dispersions are produced by transfer of the NPs from water, solvent used for their synthesis, to ChEG. When a black liquid is obtained as seen by eye, it has to be analyzed further to evaluate the quality of the dispersion and its nanostructure. Micrometric structure is analyzed by optical microscopy. If the dispersion is homogeneous at this scale, small-angle X-ray scattering (SAXS), magnetization measurements and dynamic light scattering (DLS) are used.

\subsection{Preparation of the dispersions}

Iron oxide nanoparticles are synthesized by a routine process of coprecipitation of ferric and ferrous iron ions in alkaline aqueous medium $[10,11,12]$. It produces magnetite $\left(\mathrm{Fe}_{3} \mathrm{O}_{4}\right)$ nanoparticles then fully oxidized to maghemmite $\left(\gamma \mathrm{Fe}_{2} \mathrm{O}_{3}\right)$ which is chemically stable. Final dispersed nanoparticles with a diameter of the order of $10 \mathrm{~nm}$ are obtained in acidic aqueous medium $(\mathrm{pH}=1.5)$. At this stage, the surface charge is positive $\left(\mathrm{Fe}-\mathrm{OH}_{2}^{+}\right)$and counterbalanced by $\mathrm{NO}_{3}^{-}$anions. The volume fraction of the NPs in the aqueous stock solution is $\Phi_{v}=$ $2.25 \mathrm{v} \%$, as deduced from the determination of the iron content by Flame Atomic Absorption Spectroscopy and using a density of $5 \mathrm{~g} / \mathrm{cm}^{3}$ for maghemite. Before their transfer to ChEG, the surface of the particles is covered by citrate ions, using the procedure described in [30]. Briefly, citric acid $\left(\mathrm{H}_{3} \mathrm{Cit}\right)$ is added to the acidic dispersion which leads to flocculation. $\mathrm{HNO}_{3}$ is then removed by repeated additions/ removals of $\mathrm{H}_{3}$ Cit at 0.020 $\mathrm{mol} / \mathrm{L}$. The $\mathrm{pH}$ is then increased between 7.0 and 7.5 by addition of $\mathrm{NaOH}$. The final particles are thus covered with citrate ions, $\mathrm{Na}^{+}$being the positively charged counterions. This dispersion of citrate-coated NPs will be referred to as "aqueous dispersion" from now on.

If the particles are to be transferred in the ChEG, the aqueous dispersion is first flocculated by addition of acetone, washed 3 times by mixtures of acetone:water (9:1) and once by ether. After the removal of the ether, the desired amount of ChEG is added, and the dispersion is gently heated to remove any residual organic solvent.The dispersion is then centrifuged 10 minutes at $4000 \mathrm{rpm}$ to get rid of the remaining few undispersed particles.

\subsection{Structure by optical microscopy and Small Angle X-Ray Scattering (SAXS)}

Two samples are compared, in water and in ChEG. As the aqueous systems have been extensively studied in several conditions and their colloidal stability is well controlled (see for example [31] and references therein), the dispersion in water is used as a reference. On the nanoscale, the structure is determined by SAXS. The scattered intensity I is recorded as a function of the scattering vector $\mathrm{Q}$. For very dilute NPs dispersion, $\mathrm{I}(\mathrm{Q})$ is characteristic of the shape of the scattering objects over the whole $\mathrm{Q}$ range (form factor). For more concentrated dispersions, the nanoparticles undergo colloidal interactions. The intensity at small Qs is then representative of the structure factor $\mathrm{S}(\mathrm{Q})$ of the dispersion while at high $\mathrm{Qs}$, it is still dominated by the form factor. A dilute aqueous sample is used for the determination of the form factor. It is obtained by dilution of the initial aqueous dispersion in $\mathrm{HNO}_{3} 10^{-2} \mathrm{~mol} \mathrm{~L}^{-1}$. Figure SI-2 plots the scattered intensity versus the scattering vector $\mathrm{Q}$ in absolute units. Figure 4 shows the corresponding Porod plot $I \times \mathrm{Q}^{4}$, normalized by the volume fraction $\phi_{v}$ and the contrast $\Delta \rho^{2}$, versus $\mathrm{Q}$. The volume fraction is taken from the invariant of diffusion : $\phi_{v}$ is $0.061 \mathrm{vol} \%$ for the reference sample in water and $1.13 \mathrm{vol} \%$ for the sample in ChEG. This representation $I \times \mathrm{Q}^{4}$ enhances the high Qs region and allows the comparison of the form factors. The curves in water and in ChEG are very similar, indicating that the same particles are present in both solvents.

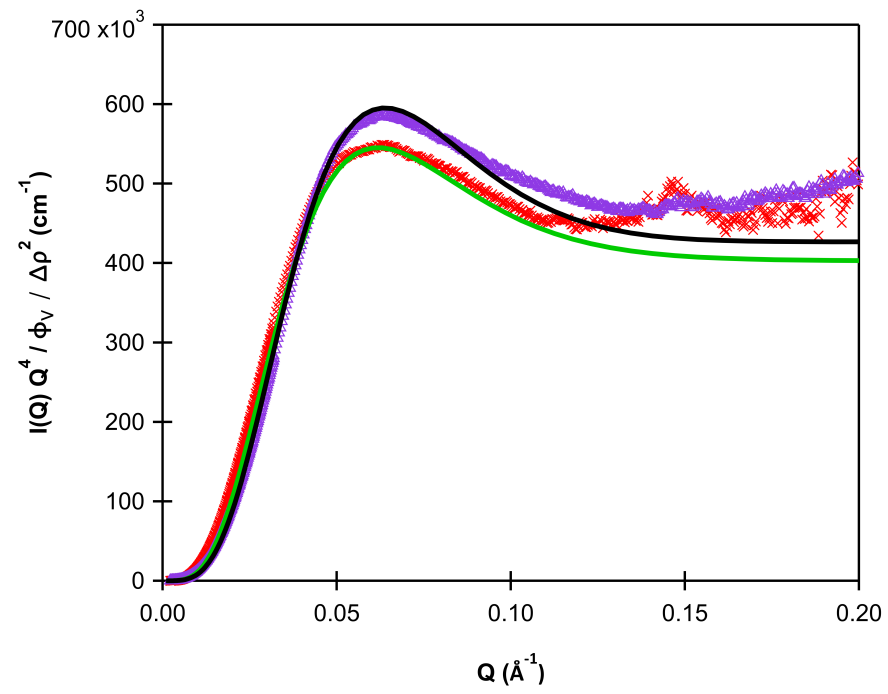

Figure 4: Porod $\left(I \times \mathrm{Q}^{4}\right)$ representation of SAXS data, in absolute unit. ( red crosses) Bare particles in aqueous $\mathrm{HNO}_{3}(\mathrm{pH}=2): \Phi_{v}=0.061 \mathrm{v} \%$ (purple triangles) Citrate-coated particles in ChEG : $\Phi_{v}=1.13 \mathrm{v} \%$. Fits were obtained with a lognormal distribution for the sizes of the particles. See text for details.

At such a low concentration of NPs in water, we can assume that the interparticle interactions are negligible. We adjust the curve $\mathrm{I}(\mathrm{Q})$ with a form factor of polydisperse spheres and a lognormal distribution. The scattering length densities $\rho$ necessary to calculate the contrast $\Delta \rho^{2}$ are known (NPs : $40.610^{10} \mathrm{~cm}^{-2}$; 
water $9.310^{10} \mathrm{~cm}^{-2}$; ChEG $10.210^{10} \mathrm{~cm}^{-2}$ ) therefore the only parameters are the median diameter $\mathrm{d}_{S A X S}$ and the polydispersity $\sigma_{S A X S}$. We use the software provided by the NIST ${ }^{2}$ to model the experimental curves [32]. The fits are performed in the $I \times \mathrm{Q}^{4}$ representation (Figure 4). Good fits are obtained in water for $\mathrm{d}_{S A X S}=6.0 \mathrm{~nm}, \sigma=0.43$ and in ChEG for $\mathrm{d}_{S A X S}=$ $6.0 \mathrm{~nm}, \sigma=0.40$. These results confirm that the size distribution is little affected by the solvent transfer. The quality of the fit is poorer at small $\mathrm{Q}$ values, indicating the presence of some aggregates in both cases (Figure SI-2).

The colloidal stability in ChEG means that some interparticular repulsions are present to counterbalance the van der Waals attraction. Information can be gained on the interactions from the scattered intensity at small Qs. The structure factor of the ChEG dispersion is calculated using the diffractogram in water (i.e., at very low concentration) as form factor : $S(Q)=\left[I_{C h E G}(Q) / \phi_{v, C h E G}\right] /\left[I_{\text {water }}(Q) / \phi_{v \text {,water }}\right]$. Indeed, we know from previous studies that interparticular interactions in water are negligeable at this $\phi$ [33]. It is reported on figure 5 . It presents an initial value $\mathrm{S}(0)$ lower that 1 and no structure peak at larger Qs, which is indicative of short range repulsions. The order of magnitude of the repulsion is similar to what was observed with the same kind of particles (maghemite covered with citrate, $\mathrm{Na}^{+}$counterions) dispersed in the ionic liquid ethylammonium nitrate [11].

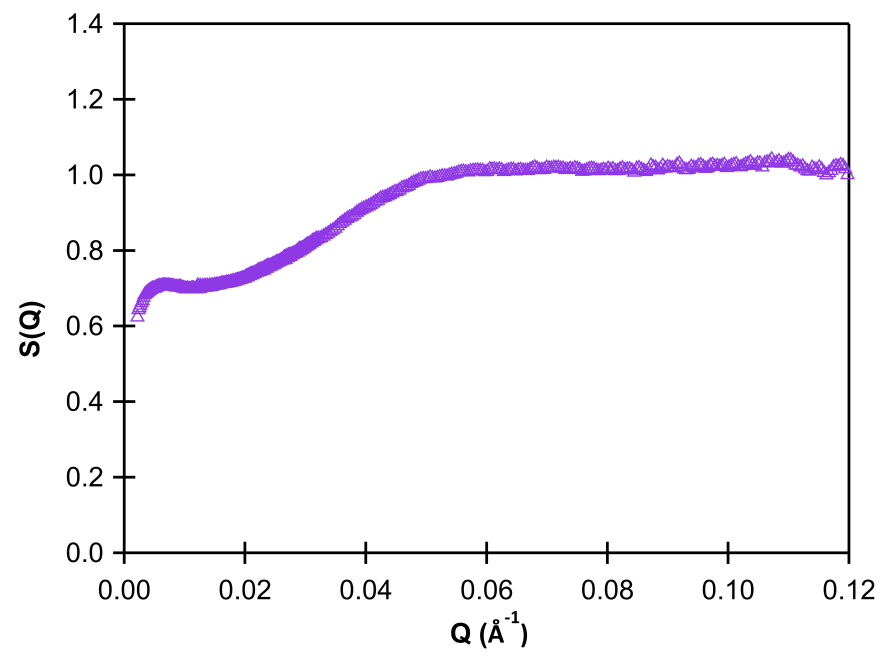

Figure 5: Structure factor $\mathrm{S}(\mathrm{Q})$ of the dispersion in ChEG at $\Phi_{v}=1.13 \mathrm{v} \%$, using the diffractogramme in water as form factor

\subsection{Magnetization measurements}

As the NPs are magnetic, the analysis of the magnetization curve of the dispersions can give information on the size distribution. However, the stability under field is checked first: both samples are stable up to $1350 \mathrm{Oe}=108 \mathrm{kA} \mathrm{m}^{-1}$ at room

\footnotetext{
${ }^{2}$ NIST : National Institute of Standards and Technology https://www.ncnr.nist.gov/programs/sans/data/red_anal.html
}

temperature. Above this threshold, the destabilization occurs in a few ten minutes. Therefore, the magnetization versus magnetic field is determined using a sequence in high fields as quick as possible in order not to destabilize the system during the measurement. The curves, plotted in Figure 6, can be fitted with the Langevin's equation coupled with a lognormal distribution of NP diameters [34]. We get here $314 \mathrm{kA} / \mathrm{m}$ for the magnetization of the material, $\mathrm{d}_{o}=7.1 \mathrm{~nm}$ and $\sigma=0.38$ for the sample in water and $\mathrm{d}_{o}=6.8 \mathrm{~nm}$ and $\sigma=0.35$ for the sample in ChEG. This distribution in ChEG is thus very close to the initial distribution in water. It means that only few large particles are removed during the transfer in $\mathrm{ChEG}$, as was already concluded from the SAXS measurements.

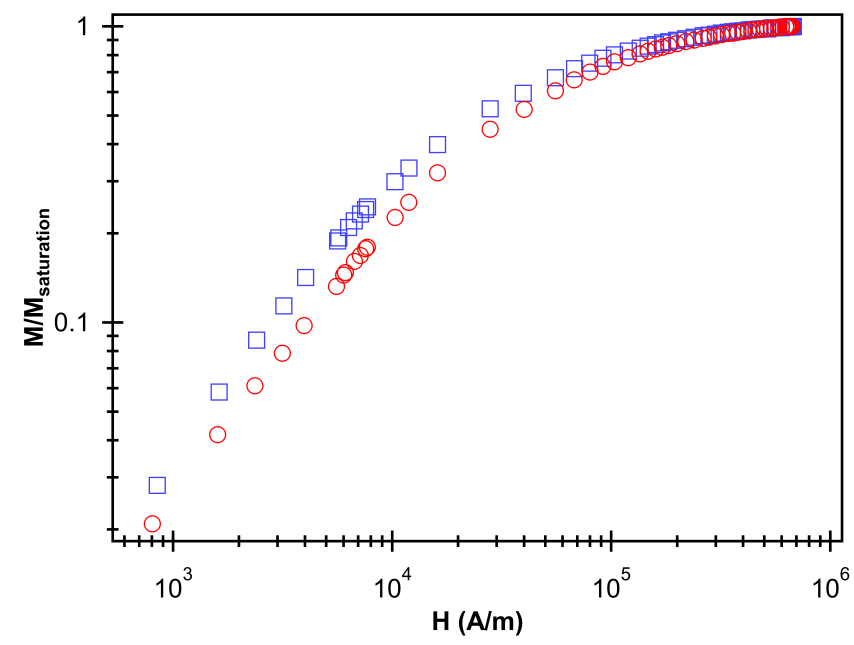

Figure 6: Normalized magnetization versus magnetic field for the samples in water (squares) and ChEG (circles).

\subsection{Dynamic Light Scattering (DLS)}

Much more accessible than SAXS, DLS is a laboratory experiment which enables studies with temperature, as well as aging and reversibility exploration. The light scattered by the sample is recorded versus time and an autocorrelation function is calculated. It measures how fast a NPs forgets its initial position thus giving access to a diffusion coefficient, which can be interpreted using appropriate models. Here, the measured intensity autocorrelation function $g_{2}(\mathrm{t})$ is converted into the field autocorrelation $g_{1}(\mathrm{t})$ function using : $g_{1}(t)=\left[g_{2}(t)\right]^{1 / 2}$ - offset. The function $g_{1}(\mathrm{t})$ of the studied samples can be fitted with a stretched exponential $g_{1}(t)=A \times \exp \left[-(t / \tau)^{\beta}\right]$ and an exponent $\beta$ close to 1 , however not perfectly, especially at long times. Henceforward, we choose (i) to fit the curves for times corresponding to $g_{1}(t)>0.1$, without taking into account the tail which is less reliable, (ii) to use a mono-exponential, i.e., $\beta=1$, in order to reduce the number of fitting parameters to only one. This corresponds to the simplest analysis, with a monodisperse population. The characteristic time $\tau$ is related to the translational diffusion coefficient $D_{t}=\left(\tau Q^{2}\right)^{-1}$. 


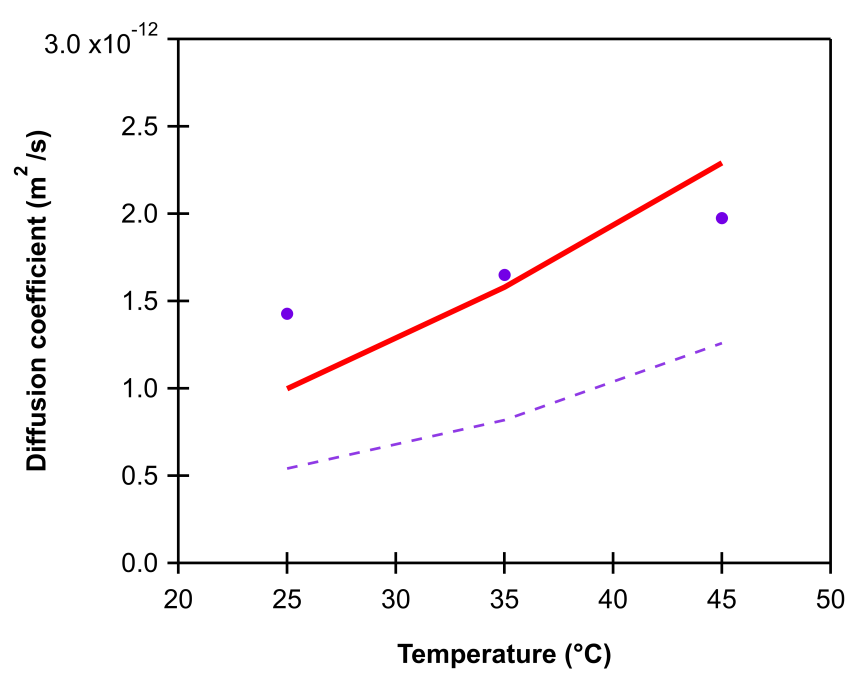

Figure 7: Diffusion coefficients $D_{t}$ from DLS measurements. Points : experimental values $D_{t, C h E G}^{\text {exp }}$ in ChEG at various T. Dashed line : calculated values $D_{t, C h E G}^{\text {calc }}$ using the measured viscosities of ChEG. Solid line : calculated values $D_{t, E G}^{c a l c}$ using the viscosity of pure EG .

The DLS measurements in ChEG are performed on the same sample as SAXS at different temperatures, $25^{\circ} \mathrm{C}, 35^{\circ} \mathrm{C}$ and $45^{\circ} \mathrm{C}$, for which macroscopic $\mathrm{ChEG}$ viscosities are known. As shown in Table 4 (and Figure 7), the diffusion coefficient $D_{t}$ increases with temperature, in a reproducible manner, even for 6 months old samples for which no evolution is seen, and going back to its value at $25^{\circ} \mathrm{C}$ after a cycle $25^{\circ} \mathrm{C}-45^{\circ} \mathrm{C}-25^{\circ} \mathrm{C}$.

\begin{tabular}{|c|c|c|c|c|}
\hline Solvent & Water & ChEG & ChEG & ChEG \\
\hline $\mathrm{T}\left({ }^{\circ} \mathrm{C}\right)$ & 25 & 25 & 35 & 45 \\
\hline $\mathrm{D}_{\text {t,exp }}$ & 1.92 & 1.43 & 1.65 & 1.97 \\
$\left(\mathrm{~m}^{2} s^{-1}\right)$ & $10^{-11}$ & $10^{-12}$ & $10^{-12}$ & $10^{-12}$ \\
\hline$\eta_{\text {macro }}$ & 0.89 & 31.6 & 21.6 & 14.5 \\
$(\mathrm{mPa} . \mathrm{s})$ & & & & \\
\hline $\mathrm{d}_{H}^{\text {app }}(\mathrm{nm})$ & 25.5 & 9.6 & 12.4 & 16.8 \\
\hline $\begin{array}{c}\eta_{\text {local }} \text { from } \\
\text { DLS (mPa.s) }\end{array}$ & & 12 & 10.7 & 9.2 \\
\hline
\end{tabular}

Table 4: Experimental diffusion coefficients $\mathrm{D}_{t, \text { exp }}$, macroscopic viscosities $\eta_{\text {macro }}$, corresponding hydrodynamic diameter $\mathrm{d}_{H}$. Viscosities $\eta_{\text {local }}$ are determined from DLS for the samples with the hypothesis that $\mathrm{d}_{H}=25.5 \mathrm{~nm}$ for all $\mathrm{T}$ in $\mathrm{ChEG}$.

\section{Discussion}

\subsection{Hydrodynamic NP diameter and local viscosity in the ChEG-FF sample}

When interparticle interaction is negligible, the translational diffusion coefficient $D_{t}$ is related to the hydrodynamic diameter $d_{H}$ by the Stokes Einstein's equation

$$
D_{t}=\frac{k_{B} T}{3 \pi \eta_{0} d_{H}}
$$

where $k_{B}$ is the Boltzmann constant, $T$ the absolute temperature, $\eta_{0}$ the local solvent shear viscosity.

Since $\mathrm{D}_{t}$ is obtained by DLS as an intensity average, the corresponding hydrodynamic diameter obtained through equation 5 is a mean value defined as $\mathrm{d}_{H}=<d^{6}>/<d^{5}>$ [35]. It can be compared to the value calculated from the lognormal distribution obtained from SAXS, $\mathrm{d}_{H}^{\text {calc }}$. In the case of the aqueous FF-sample, using for $\eta_{0}$ the macroscopic viscosity of water in Eq. 5, we obtain $\mathrm{d}_{H}^{\text {calc }}=17.0 \mathrm{~nm}$ in reasonable agreement with the DLS experimental results $d_{H}=25 \mathrm{~nm}$ (see Table 4). The DLS value is indeed somehow higher due to the presence of small aggregates (see SAXS section).

However in the case of the ChEG FF-sample, this agreement fails if $\eta_{0}$ is assimilated to the ChEG macroscopic viscosity in Eq. 5. The apparent diameter $\mathrm{d}_{H}^{a p p}$ is then found much smaller than $\mathrm{d}_{H}^{\text {calc }}$. Moreover, the size is unexpectedly temperature dependent.

Several arguments can be put forward to explain these observations. (i) A much lower polydispersity in the ChEG-FF sample could be an explanation, however not consistent with the SAXS results. (ii) Interparticle interactions could modify the apparent diffusion coefficient in the ChEG FF-sample, however the observed size decrease would correspond to a strong repulsion which is not compatible with SAXS data. (iii) A bad evaluation of the refractive index could be put forward, however the refractive indexes of liquids usually range roughly between 1.3 and 1.5 and their variation cannot explain such a large difference. (iv) The viscosity is not correct. Indeed, viscosity highly depends on the solvent composition. However a macroscopic water pollution cannot explain the results as a huge amount of water (more than $20 \%$ ) would be necessary to induce such a large decrease of the macroscopic viscosity.

The only possibility in this ChEG-FF sample, is thus a lower local viscosity around the NPs than the macroscopic ChEG viscosity. A direct comparison of the diffusion coefficients is presented in Figure 7. It plots the temperature-dependence of the experimental diffusion coefficients $D_{t, \exp }^{\operatorname{ChEG}}$ and compares them with calculated values, using the sample in water at $25^{\circ} \mathrm{C}$ as a reference : $D_{t, \text { calc }}^{\text {ChEG }}=D_{t, \text { exp }}^{\mathrm{H} 2 \mathrm{O}}(25) \times \eta_{\text {macro }}^{\mathrm{H}_{2} \mathrm{O}}(25) / \eta_{\text {macro }}^{\text {ChEG }}(T)$. The same can be done to estimate the diffusion coefficient of the particles in pure EG : $D_{t, \text { calc }}^{E G}=D_{t, \text { exp }}^{\mathrm{H2O}}(25) \times \eta_{\text {macro }}^{\mathrm{H}_{2} \mathrm{O}}(25) / \eta_{\text {macro }}^{E G}(T)$. Figure 7 shows that neither the macroscopic value of the viscosity of ChEG nor that of EG can reproduce the experimental data. A local viscosity is thus necessary around the particles to assess for the diffusion results. It can be determined through $\eta_{\text {local }}^{\mathrm{ChEG}}(T)=D_{t, \text { exp }}^{\mathrm{H} 2 \mathrm{O}}(25) / D_{t, \text { exp }}^{\mathrm{ChEG}}(T) \times \eta_{\text {macro }}^{\mathrm{H}_{2} \mathrm{O}}(25)$ (see Table 4). We observe that this local viscosity is much lower than the macroscopic one and presents a less pronounced temperature dependance.

\subsection{Analysis of the temperature-dependence and origin of the local viscosity in the ChEG-FF sample}

The analysis with the model of Eyring of the temperature dependence of the local viscosity, considering that the molar volume of the solvent is unaffected by the presence of the particles, gives contributions to the flow Gibbs energy very different from those of pure ChEG (Figure 3 and Table 3). If at 
298K the Gibbs energy value $\Delta G_{a}$ is similar in the ChEG-FF and in the complex solvent (around $19 \mathrm{~kJ} / \mathrm{mol}$ ), its splitting into enthalpy $\Delta H_{a}$ and entropy $\Delta S_{a}$ is completely modified. Indeed, $\Delta H_{a}$ is lowered from 30 to $10 \mathrm{~kJ} / \mathrm{mol}$ while $-T \Delta S_{a}$ changed sign from $-8.5 \mathrm{~kJ} / \mathrm{mol}$ to $+9 \mathrm{~kJ} / \mathrm{mol}$. This implies that the particles deeply modify the solvent organization around them, both from the solvent-solvent interaction point of view (a decrease of $\Delta H_{a}$ means that a lower number of interactions between solvents molecules must be modified for the fluid to flow) and from the entropic point of view (higher - $T \Delta S_{a}$ means that the movement of a solvent entity implies a loss of accessible configurations).

Ionic liquids are known to be strongly organized in hydrophilic and hydrophobic domains [36] and the DES made of choline chloride-urea was recently shown to be highly structured through H-bonds implying all its three components (Cl-, Cholinium, urea) [37]. By analogy with ChCl-urea, ChCl-EG can be assumed structured. In such a structured solvent, the nanoparticles can preferentially accommodate one of the solvent components and disturb the network. In this case, the viscosity experienced by the particles is not the macroscopic viscosity of the solvent, but the local viscosity of the surrounding fluid in that domain. In our case, this local viscosity is much lower than the macroscopic one, our results suggesting that the less viscous fluid, ethyleneglycol, directly surrounds the particles.

\section{Conclusion}

The density and viscosity of a mixture of choline chloride and ethylene glycol (1:3) a complex solvent close to DESs, were carefully determined from 20 to $45^{\circ} \mathrm{C}$ and for water contents between 0.2 and $4.6 \%$. The analysis of the data using the model of Eyring indicates that this highly structured solvent needs to destructurate to flow. This is quantified by the entropic contribution that is more important than the enthalpic one in the activation Gibbs energy for viscous flow. In a second part, we demonstrate that maghemite nanoparticles can be dispersed in this complex solvent thanks to a transfer from water. They are well dispersed, interacting through weak repulsions sufficient to stabilize the dispersions in zero magnetic field and under field up to 1350 Oe. This threshold could be increased using smaller particles and reduced polydispersities. These results are similar to those previously obtained in an ionic liquid, EAN and confirm that the mixture ChEG studied here behaves like an ionic liquid. Indeed, a behaviour of concentrated electrolyte solution would not allow the dispersion of NPs which would flocculate due to the screening of electrostatic interactions. The local viscosity experienced by the NPs in ChEG dispersions as well as its temperature variation indicates that the particles locally modify the solvent around the NPs. This could be a local modification of organization or of composition. The results can probably be extended to other oxide nanoparticles, with the same kind of surface groups (hydroxides).
To go further, quantitative macroscopic viscosity measurements, difficult as samples are black and hygroscopic, would be interesting to compare to microscopic viscosities. Also parallel SAXS measurements as a function of temperature would enable to get a clear picture of the local behavior of the samples.

We thank Laurent Michot for fruitful discussions and Javier Perez, our local contact on SWING beamline at SOLEIL for his help. We thank ANR TE-FLIC (grant number ANR-12PRGE-0011-01) for financial support.

\section{Supporting Information}

* Table : Viscosity and density values for each temperature $T$ and water content $x$.

* figure SI-1 : Vmol vs water content $x$, for each temperature $T$

* figure SI-2 : SAXS scattered intensity I(Q)

[1] Z. He, P. Alexandridis, Nanoparticles in ionic liquids: interactions and organization, Phys. Chem. Chem. Phys. 17 (28) (2015) 18238-18261. doi:10.1039/C5CP01620G.

URL http://xlink.rsc.org/?DOI=C5CP01620G

[2] Z. He, P. Alexandridis, Ionic liquid and nanoparticle hybrid systems: Emerging applications, Advances in Colloid and Interface Science 244 (2017) 54-70. doi:10.1016/j.cis.2016.08.004.

URL http://www.sciencedirect.com/science/article/pii/S0001868616

[3] Q. Zhang, K. D. O. Vigier, S. R. F. Jérôme, Deep eutectic solvents: syntheses, properties and applications., Chemical Society Reviews 41 (2012) 7108 .

[4] A. Abo-Hamad, M. Hayyan, M. A. AlSaadi, M. A. Hashim, Potential applications of deep eutectic solvents in nanotechnology, Chemical Engineering Journal 273 (2015) 551-567. doi:10.1016/j.cej.2015.03.091. URL http://www.sciencedirect.com/science/article/pii/S1385894715

[5] F. S. Mjalli, T. Al-Wahaibi, A. A. Al-Hashmi, Effect of nano-particles on the rheological properties of Reline, Journal of Molecular Liquids 206 (2015) 256-261. doi:10.1016/j.molliq.2015.02.040.

URL http: //www.sciencedirect.com/science/article/pii/S0167732215

[6] A. Yadav, S. Pandey, Densities and Viscosities of (Choline Chloride + Urea) Deep Eutectic Solvent and Its Aqueous Mixtures in the Temperature Range $293.15 \mathrm{~K}$ to $363.15 \mathrm{~K}$, Journal of Chemical \& Engineering Data 59 (7) (2014) 2221-2229. doi:10.1021/je5001796.

URL http://dx.doi.org/10.1021/je5001796

[7] A. P. Abbott, R. C. Harris, K. S. Ryder, Application of Hole Theory to Define Ionic Liquids by their Transport Properties, The Journal of Physical Chemistry B 111 (18) (2007) 4910-4913. doi:10.1021/jp0671998. URL http://dx.doi.org/10.1021/jp0671998

[8] F. Chen, S. Xie, X. Huang, X. Qiu, Ionothermal synthesis of Fe3o4 magnetic nanoparticles as efficient heterogeneous Fenton-like catalysts for degradation of organic pollutants with $\mathrm{H} 2 \mathrm{o} 2$, Journal of Hazardous Materials 322 (2017) 152-162. doi:10.1016/j.jhazmat.2016.02.073.

URL http://www.sciencedirect.com/science/article/pii/S0304389416

[9] X. Huang, C. Xu, J. Ma, F. Chen, Ionothermal synthesis of Cu-doped $\mathrm{Fe} 3 \mathrm{o} 4$ magnetic nanoparticles with enhanced peroxidase-like activity for organic wastewater treatment, Advanced Powder Technology 29 (3) (2018) 796-803. doi:10.1016/j.apt.2017.12.025.

URL http: //www . sciencedirect . com/science/article/pii/S0921883117

[10] R. Massart, Preparation of aqueous magnetic liquids in alkaline and acidic media, I.E.E.E., Transactions on Magnetics 2 (1981) 1247-1248.

[11] M. Mamusa, J. Sirieix-Plénet, F. Cousin, R. Perzynski, E. Dubois, V. Peyre, Microstructure of colloidal dispersions in the ionic liquid ethylammonium nitrate: influence of the nature of the nanoparticles' counterion, Journal of Physics: Condensed Matter 26 (28) (2014) 284113. doi:10.1088/0953-8984/26/28/284113.

URL http: //stacks . iop.org/0953-8984/26/i=28/a=284113?key=crossre

[12] I. T. Lucas, S. Durand-Vidal, E. Dubois, J. Chevalet, P. Turq, Surface charge density of maghemite nanoparticles: Role of electrostatics in the proton exchange, J. Phys. Chem. C 111 (50) (2007) 18568-18576. doi:10.1021/jp0743119. 
[13] M. Mamusa, J. Siriex-Plénet, F. Cousin, E. Dubois, V. Peyre, Tuning the colloidal stability in ionic liquids by controlling the nanoparticles/liquid interface, Soft Matter 10 (8) (2014) 1097. doi:10.1039/c3sm52733f.

URL http: //xlink.rsc.org/?DOI=c3sm52733f

[14] M. Mamusa, J. Sirieix-Plénet, R. Perzynski, F. Cousin, E. Dubois, V. Peyre, Concentrated assemblies of magnetic nanoparticles in ionic liquids, Faraday Discuss. 181 (2015) 193-209. doi:10.1039/C5FD00019J. URL http://xlink.rsc.org/?DOI=C5FD00019J

[15] C. Guibert, V. Dupuis, J. Fresnais, V. Peyre, Controlling nanoparticles dispersion in ionic liquids by tuning the $\mathrm{pH}$, Journal of Colloid and Interface Science 454 (2015) 105-111. doi:10.1016/j.jcis.2015.04.059. URL http://linkinghub.elsevier.com/retrieve/pii/S00219797

[16] S. Foner, Versatile and Sensitive Vibrating?Sample Magnetometer, Review of Scientific Instruments 30 (7) (1959) 548-557. doi:10.1063/1.1716679.

URL http://scitation.aip.org/content/aip/journal/rsi/30/7/

[17] B. Berkovski (Ed.), Magnetic Fluids and Applications Handbook, Begell House Inc. Publ. New York, 1996.

[18] J. Bacri, R. Perzynski, D. Salin, V. Cabuil, R. Massart, Ionic ferrofluids: a crossing of chemistry and physics, J. Magn. Magn. Mat. 85 (1990) 27-32.

[19] D. R. Lide (Ed.), CRC Handbook of chemistry and physics, 78th Edition, CRC Press, Boca Raton, Fla., 1997, oCLC: 833224623.

[20] Chemical safety information from intergovernmental organizations - choline chloride, Tech. rep., OECD Screening Information Data Set (2004).

[21] F. S. Mjalli, J. Naser, Viscosity model for choline chloride-based deep eutectic solvents, Asia-Pacific Journal of Chemical Engineering 10 (2) (2015) 273-281. doi:10.1002/apj.1873.

URL http://onlinelibrary.wiley.com/doi/10.1002/apj.1873/abstract

[22] G. I. Egorov, D. M. Makarov, A. M. Kolker, Liquid phase PVTx properties of binary mixtures of (water + ethylene glycol) in the range from 278.15 to $323.15 \mathrm{~K}$ and from 0.1 to $100 \mathrm{MPa}$. I. Experimental results, partial and excess thermodynamics properties, Fluid Phase Equilibria 344 (2013) 125-138. doi:10.1016/j.fluid.2013.01.025.

URL http://www.sciencedirect.com/science/article/pii/S0378381213000745

[23] A. K. Nain, Densities and volumetric properties of (formamide + ethanol, or 1-propanol, or 1,2-ethanediol, or 1,2-propanediol) mixtures at temperatures between $293.15 \mathrm{~K}$ and $318.15 \mathrm{~K}$, The Journal of Chemical Thermodynamics 39 (3) (2007) 462-473. doi:10.1016/j.jct.2006.07.021. URL http: //www.sciencedirect.com/science/article/pii/S0021961406001704

[24] A. K. Nain, Ultrasonic and viscometric studies of molecular interactions in binary mixtures of formamide with ethanol, 1-propanol, 1,2-ethanediol and 1,2-propanediol at different temperatures, Journal of Molecular Liquids 140 (2008) 108-116. doi:10.1016/j.molliq.2008.01.016. URL http://www.sciencedirect.com/science/article/pii/S0167732208000305

[25] Q. Abbas, L. Binder, Synthesis and characterization of choline chloride based binary mixtures, ECS Transactions 33 (7) (2010) 49-59.

[26] M. Tanaka, G. Girard, R. Davis, A. Peuto, N. Bignell, Recommended table for the density of water between $0{ }^{\circ} \mathrm{C}$ and $40{ }^{\circ} \mathrm{C}$ based on recent experimental reports, Metrologia 38 (4) (2001) 301-309.

[27] G. I. Egorov, D. M. Makarov, A. M. Kolker, Volumetric properties of the water-ethylene glycol mixtures in the temperature range 278333.15 $\mathrm{k}$ at atmospheric pressure, Russian Journal of General Chemistry 80 (8) (2010) 1577-1585. doi:10.1134/S1070363210080074

URL http://link. springer.com.chimie.gate.inist.fr/article/10.1134/S1070363210080074

[28] O. Ciocirlan, O. Iulian, O. Croitoru, , Rev Chim 61 (2010) 721-723.

URL

[29] H. Eyring, Viscosity, Plasticity, and Diffusion as Examples of Absolute Reaction Rates, The Journal of Chemical Physics 4 (4) (1936) 283-291. doi:10.1063/1.1749836.

URL http://scitation. aip.org/content/aip/journal/jcp/4/4/10.1063/1.1749836

[30] C. L. Filomeno, M. Kouyaté, V. Peyre, G. Demouchy, A. F. C. Campos, R. Perzynski, F. A. Tourinho, E. Dubois, Tuning the Solid/Liquid Interface in Ionic Colloidal Dispersions: Influence on Their Structure and Thermodiffusive Properties, The Journal of Physical Chemistry C 121 (10) (2017) 5539-5550. doi:10.1021/acs.jpcc.6b10280.

URL http://dx.doi .org/10.1021/acs.jpcc.6b10280

[31] G. Mériguet, E. Wandersman, E. Dubois, A. Cebers, J. de Andrade Gomes, G. Demouchy, J. Depeyrot, A. Robert, R. Perzynski, Magnetic fluids with tunable interparticle interaction : monitoring the under-field local structure, Magnetohydrodynamics 49 (2013) 191-201.

[32] S. R. Kline, Reduction and analysis of SANS and USANS data using
IGOR Pro, Journal of Applied Crystallography 39 (6) (2006) 895-900. doi:10.1107/S0021889806035059.

URL http://scripts .iucr .org/cgi-bin/paper?do5025

[33] E. Dubois, R. Perzynski, F. Boué, V. Cabuil, Liquid-gas transitions in charged colloidal dispersions: Small-angle neutron scattering coupled with phase diagrams of magnetic fluids, Langmuir 16 (13) (2000) 56175625 .

URL http://pubs . acs .org/doi/abs/10.1021/la000053u

[34] Magnetic Fluids and Applications Handbook.

URL http: //www . begellhouse.com/books/magnetic-fluids-and-applic

[35] P. Lindner, T. Zemb (Eds.), Neutrons, X-rays and light: scattering thods applied to soft condensed matter ; ch9, 1st Edition, NorthHolland delta series, Norh-Holland, Elsevier, Amsterdam, 2002, oCLC: 248252233

36] R. Hayes, S. Imberti, G. G. Warr, R. Atkin, Amphiphilicity determines sics 13 (8) (2011) 3237. doi:10.1039/c0cp01137a. URL http: //xlink.rsc . org/?DOI=c0cp01137a

[37] O. S. Hammond, D. T. Bowron, K. J. Edler, Liquid structure of the choline chloride-urea deep eutectic solvent (reline) from neutron diffraction and atomistic modelling, Green Chem.doi:10.1039/C5GC02914G. URL http://xlink.rsc .org/?DOI=C5GC02914G 